\title{
ON SUBHARMONICITY OF DOUBLY SUBHARMONIC FUNCTIONS
}

\author{
MAYNARD G. ARSOVE ${ }^{1}$
}

1. Introduction. Let $\Omega$ be a region in the space of two complex variables, and let $u$ be an extended real-valued function defined on $\Omega$. We shall say that $u$ is doubly subharmonic on $\Omega$ provided that $u(z, \zeta)$ is (i) subharmonic or $\equiv-\infty$ as a function of $z$ for each $\zeta$, (ii) subharmonic or $\equiv-\infty$ as a function of $\zeta$ for each $z$, and (iii) not $\equiv-\infty$. Similarly, if $u(z, \zeta)$ is harmonic in $z$ for each $\zeta$ and harmonic in $\zeta$ for each $z$, the function $u$ will be called doubly harmonic. The doubly harmonic functions on $\Omega$ are thus exactly those functions $u$ such that $u$ and $-u$ are doubly subharmonic on $\Omega$.

It is natural to ask whether doubly subharmonic functions are necessarily subharmonic. This seems still to be an open question, but V. Avanissian has shown that if $u$ is doubly subharmonic and locally bounded above, then $u$ must be subharmonic (see [2, Theorem 9 , p. $140]) .{ }^{2}$ As a consequence, every locally bounded doubly harmonic function must be harmonic.

We propose to generalize Avanissian's theorem by replacing the local majorizing constant by a summable function. Our main result is thus

THEOREM 1. If $u$ is a doubly subharmonic function on $\Omega$ admitting a locally summable majorant, then $u$ is subharmonic on $\Omega$.

This, in turn, leads to

THEOREM 2. Let $u$ be a doubly subharmonic function on $\Omega$ such that $u(z, \zeta)$ is harmonic in $z$ for each $\zeta$. If $u$ admits a locally summable minorant, then $u$ is subharmonic on $\Omega$.

As applied to doubly harmonic functions, Theorems 1 and 2 combine to yield at once

TheOREM 3. If $u$ is a doubly harmonic function on $\Omega$ admitting a locally summable majorant, then $u$ is harmonic on $\Omega$.

We shall give elementary proofs of these theorems, based on the

Received by the editors August 11, 1965 and, in revised form, December 1, 1965.

${ }^{1}$ Research supported by the Air Force Office of Scientific Research under Grant AF-AFOSR-936-65.

2 The condition that $u$ be locally bounded above was incorporated in Avanissian's definition of doubly subharmonic functions. 
use of mean-value operators. Although the details will be carried out only for functions of two 2-dimensional variables, it should be observed that the arguments remain valid in Euclidean spaces of any number of dimensions.

The assumptions as to the existence of a locally summable majorant in Theorem 1 and minorant in Theorem 2 are probably essential, but the construction of counterexamples appears to be a major task. In any event it seems unlikely that these results can be improved by techniques which rely solely on properties of the mean-value operators. On the other hand, by introducing considerations of real analyticity, P. Lelong is able to discard the majorant hypothesis in Theorem 3 (see [3, p. 561]). It would be interesting to know whether the same conclusion can be reached independently of analyticity considerations, since this would provide a proof of Hartog's theorem not requiring Hartog's lemma.

We wish to express here our appreciation to Alfred Huber, with whom the original manuscript was discussed, particularly for calling attention to the work of Avanissian, and to Victor L. Shapiro for a critical reading of the manuscript, resulting in a number of improvements.

2. Measurability of doubly subharmonic functions. In view of the fundamental role played by areal mean-value operators applied to the individual variables, we adopt the following convention. For functions of two variables the operator $A_{r}$ will denote averaging over discs of radius $r$ with respect to the first variable, and $\alpha_{\rho}$ will denote averaging over discs of radius $\rho$ with respect to the second variable. It will always be assumed that the radii are taken small enough so that the averages are defined over neighborhoods of the points under consideration. A further convention is that the superscript $n$ applied to $u$ will denote its truncate above at $n$, and a subscript $k$ will denote the truncate below at $k$.

To begin with, let us assume of $u$ merely that it is a function on $\Omega$ such that $u(z, \zeta)$ is upper semicontinuous in $\zeta$ for each $z$ and such that each of its lower truncates has the limiting mean-value properties

$$
\lim _{r \rightarrow 0} A_{r} u_{k}(z, \zeta)=\lim _{\rho \rightarrow 0} \alpha_{\rho} u_{k}(z, \zeta)=u_{k}(z, \zeta) .
$$

From the assumed upper semicontinuity it is evident that $u_{\mathbf{k}}^{n}(z, \zeta)$ is upper semicontinuous in $\zeta$, and an application of Fatou's lemma shows that the same is true of $A_{r} u_{k}^{n}(z, \zeta)$. We can thus form the integral mean $\alpha_{\rho} A_{r} u_{k}^{n}(z, \zeta)$. 
It is an easy matter to verify that the resulting iterated mean-value function $\alpha_{\rho} A_{r} u_{k}^{n}$ is continuous. In fact, starting with an upper bound $M$ for $\left|u_{k}^{n}\right|$ and observing that $M$ is also an upper bound for $\left|A_{r} u_{k}^{n}\right|$, we have

$$
\begin{aligned}
& \left|\alpha_{\rho} A_{r} u_{k}^{n}(z, \zeta)-\alpha_{\rho} A_{r} u_{k}^{n}\left(z_{0}, \zeta_{0}\right)\right| \\
& \quad \leqq \alpha_{\rho}\left|A_{r} u_{k}^{n}(z, \zeta)-A_{r} u_{k}^{n}\left(z_{0}, \zeta\right)\right|+\left|\alpha_{\rho} A_{r} u_{k}^{n}\left(z_{0}, \zeta\right)-\alpha_{\rho} A_{r} u_{k}^{n}\left(z_{0}, \zeta_{0}\right)\right| \\
& \quad \leqq M\left[\psi\left(r,\left|z-z_{0}\right|\right)+\psi\left(\rho,\left|\zeta-\zeta_{0}\right|\right)\right],
\end{aligned}
$$

where $\psi(R, d)$ stands for the Lebesgue measure of the symmetric difference of two discs of radius $R$ whose centers are separated by a distance $d$. Since the final member here tends to 0 as $z \rightarrow z_{0}$ and $\zeta \rightarrow \zeta_{0}$, the continuity of $\alpha_{p} A_{r} u_{k}^{n}$ is assured.

There is no loss of generality in taking $n>k$, and we then conclude from the representation

$$
u_{k}^{n}=u_{k}+n-u_{n}
$$

together with (2.1) that $A_{r} u_{k}^{n} \rightarrow u_{k}^{n}$ pointwise as $r \rightarrow 0$. The bounded convergence theorem thus yields $\alpha_{\rho} A_{r} u_{k}^{n} \rightarrow \alpha_{\rho} u_{k}^{n}$ as $r \rightarrow 0$, from which it is clear that $\alpha_{p} u_{\mathbf{k}}^{n}$ is measurable. Drawing once more on (2.1) and (2.2), we arrive at measurability of $u_{k}^{n}$. That $u$ must be measurable is now apparent by letting $n \rightarrow \infty$ and $k \rightarrow-\infty$.

We shall denote by $Q$ the class of all functions $u$ on $\Omega$ such that $u(z, \zeta)$ is upper semicontinuous in $\zeta$ for each $z$ and such that each of the lower truncates $u_{k}$ has the limiting mean-value properties expressed in (2.1). This class obviously contains all doubly subharmonic functions, but it is certainly much larger. It contains, for example, all functions $u$ on $\Omega$ for which $u(z, \zeta)$ is continuous in $\zeta$ for each $z$ and is representable as a difference of subharmonic functions in $z$ for each $\zeta .^{3}$

In terms of the class $Q$ the measurability property derived above can be stated as

Lemma 1. All functions $u$ in a are measurable over $\Omega .^{4}$

3. Proof of the theorems. Let us begin with Theorem 1. Focussing attention on an arbitrary point $\left(z_{0}, \zeta_{0}\right)$ of $\Omega$ and taking account of the existence of a locally summable majorant, we see that $\alpha_{\rho} A_{r} u_{k}(z, \zeta)$ is finite for $z$ on some neighborhood $S$ of $z_{0}, \zeta$ on some neighborhood $\Sigma$

${ }^{3}$ As is clear from Theorem 22, p. 344, of [1], the truncates $u_{k}$ will share these properties, and this ensures that $u$ will be in $Q$.

${ }^{4}$ Of course, this property is also at hand if "upper semicontinuous" is replaced by "lower semicontinuous" in the definition of $Q$. 
of $\zeta_{0}, \rho$ and $r$ sufficiently small, and all $k$. Moreover, the absolute con tinuity of the integral guarantees that $\alpha_{\rho} A_{r} u_{k}$ is continuous. (Here, and in what follows, the functions will be assumed to be restricted to $S \times \Sigma$.) The boundedness below of $u_{k}$ serves to justify interchanges of the order of integration in iterated integrals, and we shall use this fact without further comment. Recalling now that $A_{r} u_{k}(z, \zeta)$ is subharmonic in $z$, so that $A_{r} u_{k}(z, \zeta) \leqq A_{t} A_{r} u_{k}(z, \zeta)$, we have

$$
\alpha_{\rho} A_{r} u_{k}(z, \zeta) \leqq A_{t} \alpha_{\rho} A_{r} u_{k}(z, \zeta) .
$$

Hence, $\alpha_{\rho} A_{r} u_{k}(z, \zeta)$ is subharmonic in $z$ and (by symmetry) in $\zeta$.

Having thus established that $\alpha_{\rho} A_{r} u_{k}$ is a continuous doubly subharmonic function, we apply the areal mean-value operators once more to obtain $\alpha_{\rho}^{2} A_{r}^{2} u_{k}$. This function is easily seen to be $C^{\prime}$ and doubly subharmonic. That the first partial derivatives exist as continuous functions is, in fact, immediate from the classical formulas in 2.20 of [4]. These yield, for example,

$$
\frac{\partial}{\partial x} \alpha_{\rho}^{2} A_{r}^{2} u_{k}(z, \zeta)=\frac{1}{\pi r} \int_{0}^{2 \pi} \alpha_{\rho}^{2} A_{r} u_{k}\left(z+r e^{i \theta}, \zeta\right) \cos \theta d \theta
$$

Double subharmonicity then follows by the argument used for $\alpha_{\rho} A_{r} u_{k}$.

A repetition of this reasoning shows that the triply iterated mean $V=\alpha_{\rho}^{3} A_{r}^{3} u_{k}$ is a $C^{\prime \prime}$ doubly subharmonic function, and at this stage the procedure becomes obvious. We use the fact that $V(z, \zeta)$ is a $C^{\prime \prime}$ subharmonic function of $z(=x+i y)$ and $\zeta(=\xi+i \eta)$ separately to get $V_{x x}+V_{y y} \geqq 0$ and $V_{\xi \xi}+V_{\eta \eta} \geqq 0$, whence

$$
V_{x x}+V_{y y}+V_{\xi \xi}+V_{\eta \eta} \geqq 0 .
$$

This proves that $\alpha_{\rho}^{3} A_{r}^{3} u_{k}$ is subharmonic on some neighborhood of $\left(z_{0}, \zeta_{0}\right)$ for all $k$ and all sufficiently small $\rho$ and $r$. To deduce that $u$ is subharmonic on this neighborhood, we have only to let $\rho \rightarrow 0, r \rightarrow 0$, and $k \rightarrow-\infty$, it being observed in each case that the family of subharmonic functions under consideration is decreasing. Hence, $u$ must be subharmonic on $\Omega$, and the proof of Theorem 1 is complete.

In Theorem 2 the added hypothesis of harmonicity in $z$ results in $A_{r} u=u$, so that $\alpha_{\rho} A_{r} u=\alpha_{\rho} u$, and subharmonicity in $\zeta$ guarantees that the final term here is finite. Thus, if $\phi$ is a locally summable minorant of $u$, then $\alpha_{p} A_{r}(u-\phi)$ will exist finitely. By Fubini's theorem the nonnegative function $u-\phi$ must be locally summable, forcing $u$ to be locally summable. Theorem 2 now follows from Theorem 1 .

4. Further remarks. Whether Theorem 1 remains valid without the requirement of a locally summable majorant is still an open ques- 
tion. In this regard we wish only to point out that a technique of the sort employed by Osgood for analytic functions (see e.g. [5, Exercise 2 , p. 120]) leads to the following conclusion. Suppose that $u$ is a doubly subharmonic function on $\Omega_{1} \times \Omega_{2}$ for a pair of plane regions $\Omega_{1}, \Omega_{2}$. If $u(z, \zeta)$ is continuous in $\zeta$ for each $z$, then to any region $\Omega_{1}^{\prime}$ having compact closure on $\Omega_{1}$ there corresponds an open set $\Omega_{2}^{\prime}$ dense in $\Omega_{2}$ such that $u$ is subharmonic on $\Omega_{1}^{\prime} \times \Omega_{2}^{\prime}$.

The proof makes use of the function $M$ defined on $\Omega_{2}$ as

$$
M(\zeta)=\sup _{z \in \Omega_{1}^{\prime}} u(z, \zeta)
$$

Taking cognizance of the continuity of $u(z, \zeta)$ in $\zeta$ for each $z$, we see that $M$ must be lower semicontinuous. Since $M$ is finite-valued, this implies that it is bounded above on each neighborhood belonging to some collection of neighborhoods whose union $\Omega_{2}^{\prime}$ is dense in $\Omega_{2}$. The desired conclusion thus follows from Theorem 1.

\section{REFERENCES}

1. M. G. Arsove, Functions representable as differences of subharmonic functions, Trans. Amer. Math. Soc. 75 (1953), 327-365.

2. V. Avanissian, Fonctions plurisousharmoniques et fonctions doublement sousharmoniques, Ann. Sci. École Norm. Sup. 78 (1961), 101-161.

3. P. Lelong, Fonctions plurisousharmoniques et fonctions analytiques de variables réelles, Ann. Inst. Fourier (Grenoble) 11 (1961), 515-562.

4. T. Rad6, Subharmonic functions, Springer, Berlin, 1937.

5. S. Saks and A. Zygmund, Analytic functions, Monografie Matematyczne, Vol. 28, Polskie Towarzystwo Matematyczne, Warsaw, 1952.

UNIVERSITY OF WASHINGTON AND

Eidgenössische Technische Hochschule, Zürich 